Jato Seijas, E., Cajide Val, J., García Antelo, B. \& Zamora Rodríguez, E.T. (2018). Percepciones del profesorado universitario sobre los procesos de organización y tutorización de los Trabajos Fin de Grado. Revista Electrónica Interuniversitaria de Formación del Profesorado, 21(3), 75-91.

\title{
Percepciones del profesorado universitario sobre los procesos de organización y tutorización de los Trabajos Fin de Grado
}

Elisa Jato Seijas, José Cajide Val, Beatriz García Antelo, Elisa Teresa Zamora Rodríguez

Universidade Santiago de Compostela

\section{Resumen}

El proceso de incorporación del Trabajo Fin de Grado (TFG) en el plan de estudios de las diferentes titulaciones de grado ha propiciado el desarrollo de mecanismos organizativos y de coordinación que permiten operativizar su implementación. A su vez, ha implicado para la mayoría del profesorado un importante esfuerzo en el seguimiento y tutorización del trabajo del alumnado. Este artículo tiene por objeto explorar la percepción del profesorado de la Universidad de Santiago de Compostela (USC) sobre los componentes organizativos de la materia de TFG y el proceso de tutorización. Para ello, se ha desarrollado un estudio de corte descriptivo tipo encuesta, haciendo uso de un cuestionario elaborado ad-hoc y aplicado en formato on-line al conjunto de profesorado. En total, han participado en el estudio 282 docentes de diferentes titulaciones y áreas de conocimiento, con representación de todos los centros de la USC. Los resultados obtenidos muestran escenarios diferenciados en función de las áreas, fundamentalmente en lo referente a la carga docente, el grado de integración del TFG en el marco de los planes de estudio y su proyección hacia el desempeño profesional.

\section{Palabras clave}

Trabajo fin de grado; tutoría; profesorado; coordinación.

Contacto:

Elisa Jato Seijas

Correo electrónico: elisa.jato@usc.es 


\title{
University teachers' perceptions of the organizational processes and supervision of undergraduate dissertations
}

\begin{abstract}
The process of incorporating an undergraduate dissertation (TFG in Spanish) in the bachelor degrees' curriculum has implied that several organisational and coordination mechanisms have been created in order to allow for it to be implemented. Moreover, it has meant a considerable effort for most university teachers in terms of supervising and monitoring students' dissertations. This article aims to explore the teachers in the University of Santiago de Compostela's perceptions of the organisational components of the undergraduate dissertation's course as well as its supervision process. For this purpose, a qualitative survey study has been carried out, based on an ad-hoc on-line questionnaire targeting the entire teaching community. A total of 282 teachers across the various qualifications and knowledge areas covering all the USC centres answered the questionnaire. The results have revealed a variety of concerns affecting different areas, in particular those regarding the teaching workload, the extent to which the undergraduate dissertation is integrated in the curriculum and its relevance in the students' future professional career.
\end{abstract}

\section{Key words}

Undergraduate dissertation; tutorial; teachers; coordination.

\section{Introducción}

El contexto actual de la educación universitaria en España se ha visto indudablemente marcado por los cambios que ha supuesto el establecimiento del Espacio Europeo de Educación Superior (EEES), en lo que se refiere al diseño de un nuevo escenario en el proceso organizativo y formativo de estas enseñanzas. En este marco, el Trabajo de Fin de Grado (TFG) adquiere una importancia singular al materializar algunos de los principales elementos de renovación curriculares y didácticos.

El Real Decreto 1393/2007, que establece la ordenación de las enseñanzas oficiales de Grado y Master en España (parcialmente modificado por el RD 861/2010), configura las características fundamentales del TFG al indicar que las enseñanzas de fin de grado han de incluir, de forma generalizada y obligatoria, una materia final que tenga por objeto la elaboración y defensa de un trabajo, el cual estará orientado a la evaluación de competencias asociadas al título, y podrá tener una consideración de entre 6 y 30 créditos.

Si bien puede variar en su articulación organizativa, ya que es misión de cada universidad, en el ejercicio de su autonomía, el establecimiento de las normas relativas a la elaboración, presentación y evaluación, comparte sin embargo una serie de características clave. El desarrollo de la literatura sobre el tema (Fuertes y Balaguer, 2012; González, Agulló y González, 2013; Hernández, Moreno, Camps, Clarisó y Martínez, 2013; Mateo, Escofet, Martínez, Ventura y Vlachopoulos, 2012; Puigcerver, Martín y Renart, 2013; Rekalde, 2011; Rullán y Estapé, 2011; Sánchez, 2013; Valderrama et al., 2009; Vilardell, 2010) permite delinear algunos puntos básicos que lo identifican: 
- Es un proyecto orientado al desarrollo de una investigación, intervención o innovación en el campo profesional, que integra, desenvuelve y evalúa competencias adquiridas durante el período de docencia de grado.

- Supone un trabajo personal, ejecutado por el o la estudiante, que implica el desempeño de un rol activo y el desarrollo de la propia autonomía en el proceso de aprendizaje.

- Se presenta como un espacio propicio para que el alumnado demuestre su capacitación profesional y materialice las competencias que les harán susceptibles de insertarse en el mercado laboral.

- Tiene presente el asesoramiento académico del profesorado como facilitador en el proceso de desarrollo del conocimiento.

En el marco del sistema universitario del Reino Unido se han generado diversos estudios en relación a la experiencia del "undergraduate dissertation" (UD), los cuales hacen referencia, entre otras cuestiones, a las aportaciones que representan estos trabajos de final de la formación (Feather, Anchor y Cowton, 2014; Greenbank y Penketh, 2009; Jamieson y Gray, 2006; Malcolm, 2012; Todd, Bannister y Clegg, 2004; Todd, Smith y Bannister, 2006). Así, sitúan en primer término, el ser vehículo para la promoción de la independencia del aprendizaje. El UD fue diseñado para proporcionar al alumnado la oportunidad de trabajar de manera autónoma y poder determinar el contenido, el ritmo y el enfoque de aspectos relevantes de la tarea. Gran parte del valor percibido del UD radica en proporcionar una experiencia de aprendizaje diferente, a través de la cual el alumnado aprende a asumir la responsabilidad de alcanzar sus propios objetivos. Otro de los aspectos puestos en valor por estos autores es el relativo a las competencias transferibles relacionadas con la profesionalización, así como la potencialidad de comunicación hacia el mundo laboral. Las oportunidades que ofrece el TFG pueden ser leídas del mismo modo, en términos de autonomía y profesionalización, a la vez que se remarca su papel integrador de las competencias, tanto específicas como transversales, adquiridas a lo largo de la titulación.

El desarrollo del TFG requiere de un encaje práctico que operativice su aplicación en contextos reales. Los mecanismos y procedimientos de gestión y organización asociados a su implementación se revelan como uno de los aspectos nucleares que, junto con la tutoría, motivan reflexiones y aportaciones vinculadas a entornos diversos (universidades, facultades o titulaciones). Es el caso, entre otros, de los trabajos de Ávila y Luján (2011), Civila (2014), Elías (2012), Hernández et al. (2013), Maiztegui, Martínez, Santibáñez y Martínez (2012), Martínez y Pastor (2014), Onieva (2016), Pascual y Pascual (2012), Puigcerver, Martín y Renart (2013), Reyes-García y Díaz-Megolla (2017), Rullán, Fernández, Estapè y Márquez (2010), Sánchez (2013), Vera y Briones (2015, 2016), Vilardell (2010) y Zamora y Sánchez (2014). Estos ponen de manifiesto las dificultades y la necesidad de incidir en aspectos tales como: la preparación del profesorado tutor, la consideración del tiempo de dedicación del profesorado y del alumnado, la coordinación vertical y horizontal, el establecimiento de orientaciones claras y la difusión de la información, o los diversos procesos de asignación y tutela. Todos ellos como elementos que contribuyen a conformar un conjunto de condicionantes estructurales y de gestión en función de los cuales se articula el proceso formativo y organizativo que conlleva.

Pasado ya más de un quinquenio de experiencia en la puesta en marcha y desarrollo del proceso de ordenación del TFG, se plantea la necesidad de conocer el estado de la cuestión y explorar la valoración que se hace de su contribución. El trabajo empírico que presentamos en este artículo aborda las percepciones de uno de los principales actores 
implicados: el profesorado, tanto en su rol de tutor, como de agente involucrado en el proceso organizativo. Por lo demás, en esta investigación se ha tratado de reunir y comparar la perspectiva que desde las diferentes áreas de conocimiento se tiene en la Universidad de Santiago de Compostela (USC), lo que permite avanzar en su análisis más allá de espacios referidos a una titulación concreta o facultad determinada.

\section{Objetivos}

La información que se presenta en este artículo forma parte de una investigación más amplia cuyo objetivo general se centra en conocer la percepción del profesorado de la USC sobre el TFG. Concretamente, en este artículo se abordarán aquellos objetivos específicos relativos a los procesos de organización y tutorización. Se establecen los siguientes:

- Delimitar los componentes organizativos de la materia de trabajo fin de grado en la USC.

- Conocer la valoración del profesorado sobre el proceso de tutorización de estos trabajos.

- Explorar posibles diferencias en las dimensiones analizadas al considerar la variable área de conocimiento.

\section{Metodología}

El estudio desarrollado es de corte descriptivo tipo encuesta (Cohen y Manion, 2002; Torrado, 2004), haciendo uso para la recogida de información de un cuestionario elaborado ad-hoc y aplicado en formato on-line al conjunto de profesorado de la Universidad de Santiago de Compostela. La elección del instrumento responde tanto al propósito de la investigación como a la posibilidad de llegar al conjunto de la población y tratar de conseguir la mayor representatividad posible de la misma.

El cuestionario se sometió, de cara a analizar su validez, a la valoración de un grupo de expertos del ámbito universitario vinculados con la organización y desarrollo del TFG en las diferentes áreas de conocimiento. El resultado de este análisis dio lugar a la modificación de algunos ítems, quedando configurado finalmente por ocho bloques de contenido. En el presente trabajo sólo se analizan los tres primeros, que se corresponden con los siguientes:

- Bloque I. Datos de identificación: engloba la información referente a las variables sexo, edad, área de conocimiento, centro y número de trabajos tutorizados y evaluados como miembro de un tribunal.

- Bloque II. Relevancia, organización y tutorización: integra cuestiones referidas a aspectos organizativos y de coordinación de la materia en el centro, el proceso de tutorización por parte de los/as docentes y la relevancia para la formación del alumnado.

- Bloque III. Modalidades y formatos del TFG: comprende cuestiones vinculadas al uso y relevancia de sus diferentes modalidades en el centro.

El formato de las preguntas que conforman el instrumento son de diferente tipología: preguntas cerradas con una o varias opciones de respuesta e ítems de escala de construcción tipo Likert con 5 alternativas (de 1-nada a 5-mucho). El cálculo de la consistencia interna de las escalas del instrumento se ha realizado mediante el coeficiente alpha de Cronbach, obteniendo un valor de 0,74. 
El análisis descriptivo e inferencial de los datos se ha efectuado a través del paquete estadístico SPSS versión 20.0. En el análisis descriptivo se han extraído las medidas de tendencia central y dispersión, mientras que en el análisis comparativo se han aplicado las pruebas estadísticas ANOVA y Scheffé.

\section{Participantes}

En el estudio han participado un total de 282 docentes de la Universidad de Santiago de Compostela, de los cuales el $53,9 \%$ son hombres y el $46,1 \%$ mujeres. Respecto a la edad, el $53,2 \%$ tiene más de 50 años, el 40,8\% está entre los 41 a 50 años y sólo el 6,1\% tiene menos de 40. El $9,9 \%$ ha ejercido como coordinador/a del TFG en alguna de las titulaciones de su centro. Por áreas de conocimiento, el mayor porcentaje se sitúa en Ciencias Sociales y Jurídicas (31,9\%), seguido de Ciencias de la Salud (20,2\%), Ciencias Experimentales (19,5\%), Humanidades (17,7\%) y Enseñanzas Técnicas (10,6\%). La mayoría de integrantes de la muestra pertenece al Campus de Santiago (79,8\%) frente al 20,2\% que imparte docencia en el Campus de Lugo (tabla 1).

Tabla 1.

Descripción de la muestra

\begin{tabular}{|c|c|c|c|}
\hline & & Frecuencia & $\%$ \\
\hline \multirow[t]{2}{*}{ Sexo } & Hombre & 152 & 53,9 \\
\hline & Mujer & 130 & 46,1 \\
\hline \multirow[t]{3}{*}{ Edad } & Menos de 40 años & 17 & 6,1 \\
\hline & De 41 a 50 años & 115 & 40,8 \\
\hline & Más de 50 años & 150 & 53,2 \\
\hline \multirow[t]{2}{*}{ Coordinador de TFG } & $\mathrm{Si}$ & 28 & 9,9 \\
\hline & No & 254 & 90,1 \\
\hline \multirow{5}{*}{$\begin{array}{l}\text { Área de } \\
\text { conocimiento }\end{array}$} & Ciencias Sociales y Jurídicas & 90 & 31,9 \\
\hline & Ciencias de la Salud & 57 & 20,2 \\
\hline & Ciencias Experimentales & 55 & 19,5 \\
\hline & Humanidades & 50 & 17,7 \\
\hline & Enseñanzas Técnicas & 30 & 10,6 \\
\hline \multirow[t]{2}{*}{ Campus } & Santiago & 225 & 79,8 \\
\hline & Lugo & 57 & 20,2 \\
\hline
\end{tabular}


Están además representados todos los centros docentes de la USC (25), destacando la participación del profesorado de la Facultad de Filología (9,9\%), Facultad de Ciencias de la Educación (9,2\%), Escuela Politécnica Superior (6,7\%), Facultad de Psicología (6,0\%), Escuela Politécnica Superior de Ingeniería (5,3\%), Facultad de Ciencias Económicas y Empresariales $(5,3 \%)$ y la Facultad de Derecho (5,3\%), entre otros.

\section{Resultados}

A continuación se exponen los principales resultados obtenidos, tomando como dimensiones para el análisis los componentes organizativos y el proceso de tutorización y seguimiento del TFG.

\section{Componentes organizativos del TFG}

En el marco del presente estudio, una de las dimensiones esenciales era explorar la percepción del profesorado sobre aspectos organizativos como la carga docente, la tipología de trabajos y su organización en el seno de los nuevos planes de estudio.

Respecto a la carga docente en dicha materia, la mayoría de profesorado encuestado $(60,6 \%)$ indica que durante el curso académico 2014-2015 sólo ha tutorizado de 1 a 3 estudiantes de TFG, seguido del $24,1 \%$ que supervisó el trabajo de entre 4 y 6 alumnos. Con una mayor carga docente se sitúa el $6,4 \%$ de la muestra que señala haber realizado el seguimiento de 7 a 9 trabajos $(5,3 \%)$ e incluso 10 o más (1,1\%) (tabla 2 ). Al analizar esta realidad por área de conocimiento se constata que mientras las áreas de Enseñanzas Técnicas y Ciencias Experimentales son las que tienen una menor ratio de alumnos/as tutorizados por docente, el profesorado del área de Ciencias Sociales y Jurídicas es el que, por el contrario, asume un mayor encargo docente en la materia.

Tabla 2.

Trabajos fin de grado tutorizados por docente (curso 2014-2015) (\%)

\begin{tabular}{llllll}
\hline & Ninguno & $\mathbf{1}$ a 3 & 4 a 6 & 7 a 9 & 10 o más \\
\hline C. Sociales y Jurídicas & 3,3 & 40,4 & 43,3 & 11,1 & 2,2 \\
\hline Humanidades & 8,0 & 66,0 & 22,0 & 4,0 & - \\
\hline Enseñanzas Técnicas & 3,3 & 80,0 & 13,3 & 3,3 & - \\
\hline C. Experimentales & 18,2 & 74,5 & 7,3 & - & - \\
\hline C. de la Salud & 12,3 & 64,9 & 17,5 & 3,5 & 1,8 \\
\hline Total & 8,9 & 60,6 & 24,1 & 5,3 & 1,1 \\
\hline
\end{tabular}

Otro aspecto que requiere una especial consideración es la participación en la evaluación de estos trabajos. A este respecto, cabe indicar que casi un tercio de los/as encuestados/as $(32,6 \%)$ señalan no haber formado parte de ningún tribunal durante el curso 2014-2015, mientras el $67,4 \%$ restante sí lo ha hecho, aunque en diferente grado (tabla 3). De nuevo se establecen diferencias entre las áreas de conocimiento. En el caso del profesorado de las titulaciones de Ciencias Experimentales, más de la mitad (60\%) señala no haber estado en 
ningún tribunal de TFG durante el curso 2014-2015, seguido del área de Ciencias de la Salud, en la que en un $43,9 \%$ indica esto mismo. Por el contrario, es nuevamente en el área de Ciencias Sociales y Jurídicas en la que los/as docentes encuestados/as manifiestan haber participado en la evaluación de un mayor número de TFG.

Tabla 3.

Participación en tribunales de evaluación de TFG (curso 2014-2015) (\%)

\begin{tabular}{lllll}
\hline & Ninguno & $\mathbf{1}$ a $\mathbf{5}$ & $\mathbf{6}$ a 10 & Más de 10 \\
\hline C. Sociales y Jurídicas & 10,0 & 36,7 & 35,6 & 17,8 \\
\hline Humanidades & 32,0 & 30,0 & 18,0 & 20,0 \\
\hline Enseñanzas Técnicas & 30,0 & 43,3 & 13,3 & 13,3 \\
\hline C. Experimentales & 60,0 & 18,2 & 14,5 & 7,3 \\
\hline C. de la Salud & 43,9 & 31,6 & 17,5 & 7,0 \\
\hline Total & 32,6 & 31,6 & 22,3 & 13,5 \\
\hline
\end{tabular}

En cuanto a la tipología de trabajos de fin de grado, la formulación establecida en el reglamento de la USC (2016) es lo suficientemente amplia como para que, en el marco de las directrices de cada centro, se desarrollen diferentes modalidades que se aglutinan en tres grandes tipos: investigación documental, investigación empírica y diseño de un proyecto profesional.

La investigación documental es la que tiene, a juicio del profesorado, un mayor peso en los centros y por tanto una media más elevada $(3,69)$, seguida de la investigación empírica $(3,06)$; mientras que el diseño de proyectos profesionales es menor $(2,55)$. Por otro lado, al solicitar al profesorado su valoración sobre la relevancia de estas tres modalidades para la formación del alumnado, se constata que en todas ellas se obtiene una media similar (gráfico 1).

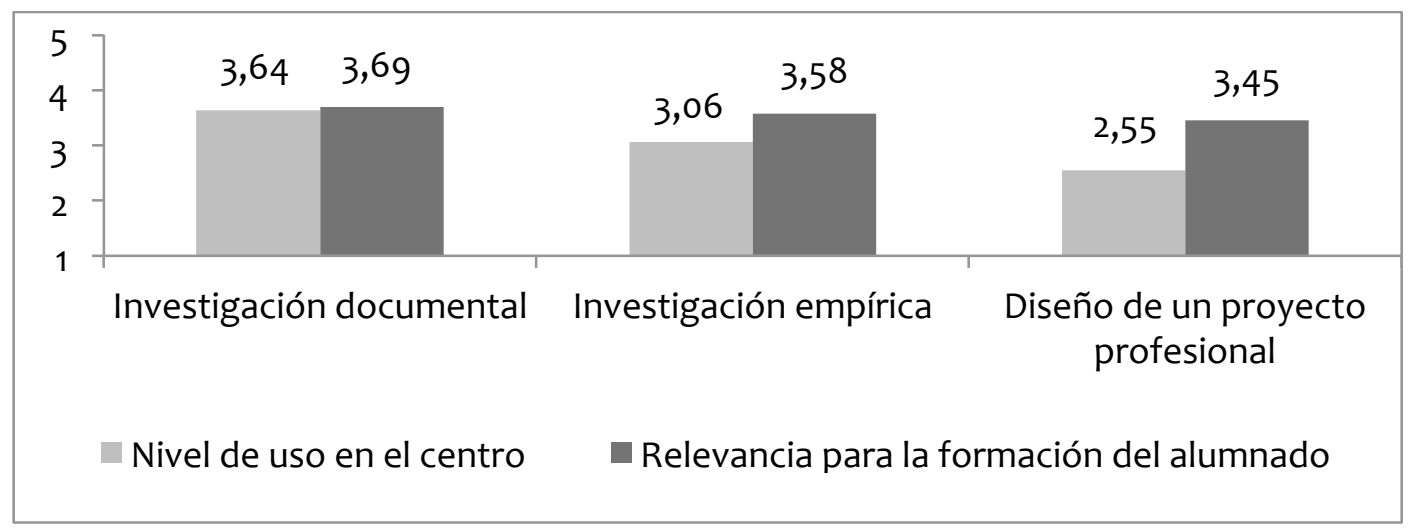

Gráfico 1. Modalidades de TFG: nivel de uso y relevancia para la formación del alumnado (media) 
En un análisis por área de conocimiento se establece la existencia de diferencias significativas tanto en el nivel de uso como en la relevancia que el profesorado otorga a la investigación documental y al diseño de un proyecto profesional (tabla 4).

Tabla 4.

Modalidades de TFG: comparativa por áreas de conocimiento

\begin{tabular}{|c|c|c|c|c|c|c|c|c|}
\hline \multicolumn{2}{|c|}{ ÍTEMS } & \multicolumn{5}{|c|}{ MEDIA } & \multicolumn{2}{|c|}{ ANOVA } \\
\hline & & $\begin{array}{l}\text { C. Soc. } \\
\text { y Juríd. }\end{array}$ & Huma. & $\begin{array}{l}\text { E. } \\
\text { TécniC. }\end{array}$ & $\begin{array}{l}\text { C. } \\
\text { Exper. }\end{array}$ & $\begin{array}{l}\text { C. } \\
\text { Salud }\end{array}$ & $\mathrm{F}$ & Sig. \\
\hline \multirow{2}{*}{$\begin{array}{l}\text { Investigación } \\
\text { documental }\end{array}$} & Uso & 3,77 & 3,90 & 2,30 & 3,44 & 4,12 & 20,361 &, $000 * *$ \\
\hline & Relevancia & 3,68 & 4,10 & 2,80 & 3,76 & 3,74 & 7,629 &, $000 * *$ \\
\hline \multirow{2}{*}{$\begin{array}{l}\text { Investigación } \\
\text { empírica }\end{array}$} & Uso & 3,02 & 3,02 & 2,73 & 3,44 & 2,98 & 2,212 & ,068 \\
\hline & Relevancia & 3,57 & 3,56 & 3,27 & 3,75 & 3,63 & ,841 &, 500 \\
\hline \multirow{2}{*}{$\begin{array}{l}\text { Diseño de un } \\
\text { proyecto }\end{array}$} & Uso & 2,69 & 1,90 & 4,43 & 2,11 & 2,32 & 29,245 &, $000 * *$ \\
\hline & Relevancia & 3,68 & 2,92 & 4,67 & 2,98 & 3,35 & 11,353 &, $000 * *$ \\
\hline
\end{tabular}

$* \mathrm{p} \leq, 05 \quad * * \mathrm{p} \leq, 01$

Más concretamente, los resultados de la prueba de Scheffé ponen de manifiesto diferencias estadísticamente significativas entre las Enseñanzas Técnicas y las restantes áreas. Así, mientras los trabajos de tipo documental tienen poca presencia en esta área (media=2,30), adquieren un mayor peso en Ciencias de la Salud (media=4,12), Humanidades (media=3,90) y Ciencias Sociales y Jurídicas (media=3,77). La relevancia otorgada a la investigación documental para la formación del alumnado sigue la misma tendencia, siendo mucho menor la concedida por el profesorado de titulaciones de Enseñanzas Técnicas frente al de las restantes áreas de conocimiento.

Esta valoración se invierte en los TFG que suponen el diseño de un proyecto profesional. Así, la mayor presencia se sitúa en este caso en el área de Enseñanzas Técnicas (media=4,43) frente a otras áreas cuyo nivel de uso es mucho menor, tales como Humanidades (media=1,90) y Ciencias Experimentales (media=2,11). De igual modo, se observa que la importancia concedida a esta modalidad de proyecto profesional es mucho mayor en el área de Enseñanzas Técnicas (media=4,67), aunque también se reconozca su relevancia para la formación del alumnado en otras áreas como Ciencias Sociales y Jurídicas ( media $=3,68$ ) y Ciencias de la Salud (media=3,35).

Por lo que se refiere a la percepción del profesorado en torno a los procesos de organización y coordinación del TFG (tabla 5), los resultados obtenidos ponen de manifiesto la conformidad del profesorado con cuestiones organizativas como el reglamento de la materia en el centro, que consideran que establece con claridad el procedimiento a seguir (media=4,02), así como con la labor de coordinación desarrollada (media=3,61). No obstante, se observa una mayor diversidad en relación a otras cuestiones estructurales. Tal es el caso de la valoración sobre el número de créditos asignados al TFG, siendo claramente insuficientes para las áreas de Ciencias Sociales y Jurídicas y Ciencias de la Salud. Dado que 
el número de créditos del TFG puede variar de unas titulaciones a otras, es lógico encontrar esta heterogeneidad en las respuestas aportadas.

Igualmente heterogéneas son las respuestas respecto a la consideración de si el TFG prepara para el ejercicio profesional, si el proceso de implementación de la materia cumple las expectativas en cuanto a calidad y si el TFG abre posibilidades para la innovación y la transferencia del conocimiento a la sociedad (tabla 5). El análisis de varianza realizado pone de manifiesto diferencias estadísticamente significativas en estos ítems según el área de conocimiento. Asi mismo, la prueba Scheffé identifica entre cuáles de ellas se da esta diferencia, siendo el profesorado de Enseñanzas Técnicas el que apoya en mayor medida estas afirmaciones, frente al resto de áreas, que le otorgan una valoración mucho menor.

Tabla 5.

Percepción del profesorado sobre la organización y coordinación del TFG en el centro: comparativa por áreas de conocimiento

\begin{tabular}{|c|c|c|c|c|c|c|c|c|}
\hline \multirow[t]{2}{*}{ ÍTEMS } & \multicolumn{6}{|c|}{ MEDIAS } & \multicolumn{2}{|c|}{ ANOVA } \\
\hline & $\begin{array}{l}\text { C. Soc. } \\
\text { y Jur. }\end{array}$ & Hum. & $\begin{array}{l}\text { E. } \\
\text { Técn. }\end{array}$ & $\begin{array}{l}\text { C. } \\
\text { Exp. }\end{array}$ & $\begin{array}{l}\text { C. } \\
\text { Salud }\end{array}$ & Total & $\mathrm{F}$ & Sig. \\
\hline $\begin{array}{l}\text { El número de créditos } \\
\text { asignados al TFG } \\
\text { concuerda con la } \\
\text { relevancia que se le } \\
\text { concede en la práctica. }\end{array}$ & 2,58 & 3,38 & 3,10 & 3,23 & 2,68 & 2,92 & 5,119 &, $001 * *$ \\
\hline $\begin{array}{l}\text { El reglamento del TFG de } \\
\text { su centro establece con } \\
\text { claridad el procedimiento } \\
\text { a seguir. }\end{array}$ & 3,90 & 4,18 & 4,33 & 4,07 & 3,86 & 4,02 & 1,869 & ,116 \\
\hline $\begin{array}{l}\text { La labor de coordinación } \\
\text { en su centro favorece el } \\
\text { desarrollo organizativo } \\
\text { del TFG. }\end{array}$ & 3,55 & 3,65 & 3,93 & 3,56 & 3,52 & 3,61 & ,822 &, 512 \\
\hline $\begin{array}{l}\text { El proceso de } \\
\text { implementación de la } \\
\text { materia del TFG cumple } \\
\text { las expectativas en } \\
\text { cuanto a calidad. }\end{array}$ & 2,55 & 2,62 & 3,46 & 3,07 & 2,70 & 2,79 & 5,367 &, $000 * *$ \\
\hline $\begin{array}{l}\text { El TFG prepara para el } \\
\text { ejercicio profesional. }\end{array}$ & 2,89 & 2,71 & 4,23 & 3,00 & 2,86 & 3,02 & 11,743 &, $000 * *$ \\
\hline $\begin{array}{l}\text { El TFG abre posibilidades } \\
\text { para la innovación y la } \\
\text { transferencia del } \\
\text { conocimiento a la } \\
\text { sociedad. }\end{array}$ & 2,64 & 2,50 & 3,57 & 2,50 & 2,55 & 2,67 & 4,991 &, $001 * *$ \\
\hline
\end{tabular}




\section{El proceso de tutorización y seguimiento del TFG}

Respecto al proceso de asignación de tutores/as (tabla 6), los/as encuestados/as expresan que el criterio que debería tener un mayor peso son las líneas temáticas o de investigación del profesorado (media $=4,44$ ) y las preferencias del alumno/a (media $=4,14$ ), mientras que conceden una menor importancia al expediente académico (media=3,13).

Tabla 6.

Importancia concedida a los criterios para la asignación de tutores/as

\begin{tabular}{|c|c|c|c|c|c|}
\hline \multirow[t]{2}{*}{ ÍTEMS } & \multicolumn{2}{|c|}{$\begin{array}{l}\text { Estadísticos } \\
\text { descriptivos }\end{array}$} & \multicolumn{3}{|c|}{ Porcentajes } \\
\hline & Media & $\begin{array}{l}\text { Desv. } \\
\text { típica }\end{array}$ & $\begin{array}{l}\text { Nada o } \\
\text { poco }\end{array}$ & Regular & $\begin{array}{l}\text { Bastante } \\
\text { o mucho }\end{array}$ \\
\hline $\begin{array}{l}\text { Expediente académico } \\
\text { del alumno/a }\end{array}$ & 3,13 & 1,389 & 35,6 & 18,3 & 46,0 \\
\hline $\begin{array}{l}\text { Preferencias } \\
\text { alumno/a }\end{array}$ & 4,14 & ,999 & 6,5 & 13,6 & 80,0 \\
\hline $\begin{array}{l}\text { Líneas temáticas de } \\
\text { los/as tutores/as }\end{array}$ & 4,44 & ,904 & 4,7 & 5,8 & 89,6 \\
\hline
\end{tabular}

El intenso trabajo de tutorización y seguimiento que exige al profesorado el desarrollo de los trabajos fin de grado también ha sido objeto de análisis (tabla 7). Al respecto, la mayoría de encuestados/as confirman que la carga de trabajo real supera la asignada en el Plan de Organización Docente (POD) (media=4,69), teniendo además dificultades para cumplir con el calendario establecido (media=3,65). Para realizar el seguimiento de estos trabajos, el profesorado indica mayoritariamente que programa un calendario de tutorías con el alumnado (media=3,91) y que su experiencia en el TFG lo ha llevado a adoptar mejoras en el proceso de tutorización (media=3,69).

Por otro lado, la formación previa del alumnado condiciona en gran medida el desarrollo de este proceso de seguimiento. En efecto, en términos generales, el profesorado coincide en que la realización del TFG integra la aplicación de diversas metodologías y herramientas trabajadas en las materias (media $=3,47$ ), aunque discrepa en que el alumnado tenga competencias suficientes para enfrentar con autonomía la elaboración del TFG (media=2,59). Así pues, si bien parece no haber muchos centros que oferten actividades formativas específicas para el alumnado al inicio del TFG (media=2,77), la gran mayoría aboga porque se potencie el desarrollo de acciones comunes de formación sobre temáticas clave como la búsqueda documental, metodología, etc. (media=4,29). Además, igual que ocurre con la carga docente, muchos/as de los encuestados/as coinciden en que la carga de trabajo autónomo del alumnado supera la establecida en el programa (media=3,23).

Los resultados del análisis de varianza muestran diferencias estadísticamente significativas entre las medias por áreas de conocimiento en cinco de los diez ítems (tabla 7). El profesorado de Enseñanzas Técnicas es el que considera en mayor grado que el alumnado 
posee las competencias necesarias para enfrentar con autonomía la elaboración del TFG y que su realización integra la aplicación de diversas metodologías y herramientas trabajadas en el conjunto de las materias. Del mismo modo, son los/as docentes de Ciencias Sociales y Jurídicas los/as que muestran un mayor interés en incluir acciones formativas comunes para todo el alumnado al inicio del TFG, siendo menor la necesidad percibida sobre este aspecto por el profesorado de Enseñanzas Técnicas y Ciencias Experimentales (tabla 7).

Tabla 7.

Tutorización y seguimiento de los TFG

\begin{tabular}{|c|c|c|c|c|c|c|c|c|}
\hline \multirow[t]{2}{*}{ ÍTEMS } & \multicolumn{6}{|c|}{ MEDIAS } & \multicolumn{2}{|c|}{ ANOVA } \\
\hline & $\begin{array}{l}\text { C. Soc. } \\
\text { y Jur. }\end{array}$ & Hum. & $\begin{array}{l}\text { E. } \\
\text { Técn. }\end{array}$ & $\begin{array}{l}\text { C. } \\
\text { Exp. }\end{array}$ & $\begin{array}{l}\text { C. } \\
\text { Salud }\end{array}$ & Total & $\mathrm{F}$ & Sig. \\
\hline $\begin{array}{l}\text { La carga de trabajo real } \\
\text { que supone para el } \\
\text { profesorado a la } \\
\text { tutorización del TFG } \\
\text { supera la carga asignada } \\
\text { en el POD. }\end{array}$ & 4,87 & 4,67 & 4,46 & 4,62 & 4,61 & 4,69 & 1,840 & 121 \\
\hline $\begin{array}{l}\text { El profesorado tiene } \\
\text { dificultades para cumplir } \\
\text { con el calendario } \\
\text { establecido. }\end{array}$ & 3,77 & 3,88 & 3,41 & 3,35 & 3,64 & 3,65 & 2,081 & ,084 \\
\hline $\begin{array}{l}\text { El profesorado asume la } \\
\text { tutorización de trabajos } \\
\text { no vinculados con su } \\
\text { especialidad. }\end{array}$ & 3,03 & 2,77 & 2,70 & 2,26 & 2,88 & 2,78 & 2,488 &, $044^{*}$ \\
\hline $\begin{array}{l}\text { En su papel como tutor/a } \\
\text { programa un calendario } \\
\text { de tutorías con el } \\
\text { alumnado. }\end{array}$ & 4,08 & 3,82 & 4,03 & 3,47 & 4,07 & 3,91 & 2,802 &, $026 *$ \\
\hline $\begin{array}{l}\text { Su experiencia en el TFG } \\
\text { lo llevó a adoptar mejoras } \\
\text { en el proceso de } \\
\text { tutorización. }\end{array}$ & 3,92 & 3,58 & 3,57 & 3,46 & 3,72 & 3,69 & 1,659 & 160 \\
\hline $\begin{array}{l}\text { La realización del TFG } \\
\text { integra la aplicación de } \\
\text { diversas metodologías y } \\
\text { herramientas presentadas } \\
\text { en las materias. }\end{array}$ & 3,30 & 3,57 & 4,20 & 3,39 & 3,32 & 3,47 & 4,409 &, $002 * *$ \\
\hline $\begin{array}{l}\text { El alumnado tiene } \\
\text { competencias para } \\
\text { enfrentar con autonomía } \\
\text { la elaboración del TFG. }\end{array}$ & 2,50 & 2,46 & 3,37 & 2,58 & 2,42 & 2,59 & 5,871 &, $000 * *$ \\
\hline
\end{tabular}




\begin{tabular}{|c|c|c|c|c|c|c|c|c|}
\hline $\begin{array}{l}\text { La carga de trabajo } \\
\text { autónomo del alumnado } \\
\text { supera la establecida en el } \\
\text { programa. }\end{array}$ & 3,20 & 3,23 & 3,39 & 3,29 & 3,13 & 3,23 & ,288 & ,886 \\
\hline $\begin{array}{l}\text { Desde su centro se oferta } \\
\text { alguna acción formativa } \\
\text { específica para el } \\
\text { alumnado al inicio del } \\
\text { TFG. }\end{array}$ & 2,82 & 2,60 & 2,46 & 2,92 & 2,85 & 2,77 & ,763 &, 550 \\
\hline $\begin{array}{l}\text { Sería de interés incluir } \\
\text { acciones formativas } \\
\text { comunes para todo el } \\
\text { alumnado al inicio del TFG } \\
\text { (búsqueda documental, } \\
\text { metodología, etc.) }\end{array}$ & 4,57 & 4,27 & 3,80 & 4,00 & 4,43 & 4,29 & 4,438 &, $002 * *$ \\
\hline
\end{tabular}

$* \mathrm{p} \leq, 05 \quad * * \mathrm{p} \leq, 01$

La normativa de la USC sobre la elaboración y defensa de los trabajos de fin de grado establece la posibilidad de que estos se realicen en el marco de convenios con empresas u otras instituciones, por lo que se ha considerado esencial indagar en su presencia real en los centros. Al respecto, del conjunto de la muestra, sólo el 12,4\% de encuestados/as afirma haber tutorizado algún TFG de estas características. No obstante, analizando los resultados por áreas de conocimiento, se constata que la mayoría de estas experiencias se sitúan en el área de Enseñanzas Técnicas, donde más de la mitad del profesorado ha dirigido trabajos similares (53,3\%), contrariamente a lo que ocurre en las restantes áreas, cuya incidencia es mucho menor (tabla 8).

Tabla 8.

Porcentaje de profesorado que tutorizó algún TFG en el marco del convenio con alguna empresa o institución

\begin{tabular}{lcccccc}
\hline & $\begin{array}{l}\text { C. Sociales } \\
\text { y Jurídicas }\end{array}$ & Humanid. & E.Técnicas & C. Experim. & C. Salud & Total \\
\hline SI & 6,7 & 2,0 & 53,3 & 9,1 & 12,3 & 12,4 \\
\hline NO & 93,3 & 98,0 & 46,7 & 90,9 & 87,7 & 87,6 \\
\hline
\end{tabular}

En aquellos casos en que han participado, la valoración de la experiencia es mayoritariamente alta $(22,9 \%)$ o muy alta $(48,6 \%)$, así como la importancia concedida a la formación del alumnado (37,1\% señala bastante y 54,3\% mucha importancia) (tabla 9). 
Tabla 9.

Experiencia como tutor/a de un trabajo en colaboración con una empresa/institución (\%)

\begin{tabular}{lll}
\hline \multirow{2}{*}{$\begin{array}{l}\text { Valoración de la experiencia como } \\
\text { tutor/a }\end{array}$} & Muy alta & 48,6 \\
\cline { 2 - 3 } & Alta & 22,9 \\
\cline { 2 - 3 } & Regular & 17,1 \\
\cline { 2 - 3 } & Baja & 8,6 \\
\cline { 2 - 3 } & Muy baja & 2,9 \\
\hline Importancia concedida a la & Mucha & 54,3 \\
\cline { 2 - 3 } formación del alumnado & Bastante & 37,1 \\
\cline { 2 - 3 } & Regular & - \\
\cline { 2 - 3 } & Poca & 5,7 \\
\cline { 2 - 3 } & Ninguna & 2,9 \\
\hline
\end{tabular}

\section{Discusión y conclusiones}

La inclusión del TFG en el plan de estudios de las diferentes titulaciones de grado ha motivado la creación de documentos y estructuras organizativas que permiten operativizar su implementación: las normativas de cada universidad, las guías o normas específicas de los diferentes centros, la creación de organismos como comisiones, coordinaciones, etc. Estos componentes organizativos son valorados positivamente por el profesorado encuestado, lo que muestra un buen funcionamiento de las dinámicas de gestión y coordinación de esta materia en los centros de la USC. Ahora bien, las características específicas de cada facultad en cuanto al número de alumnado, el número de grados, la carga docente del profesorado en la materia, el número de créditos asignados al TFG, etc. dibujan escenarios diferentes que condicionan la labor realizada por el profesorado tutor.

En el marco de la presente investigación, las diferencias tienen lugar fundamentalmente entre el área de Enseñanzas Técnicas y las restantes áreas de conocimiento. La primera de ellas se sitúa en la carga docente. Así, si bien el conjunto del profesorado considera que el volumen de trabajo real en la materia supera la asignada en POD, en línea con lo reconocido por otros estudios previos (Castro, Prats y Arànega, 2013), en nuestro caso se constata además que esta realidad se hace más acusada en las áreas de Ciencias Sociales y Jurídicas, que asumen la supervisión de un mayor número de alumnado.

Por otro lado, la segunda de las diferencias se relaciona con el grado de integración del TFG en el marco de los planes de estudio y su proyección hacia el desempeño profesional. Así, es el profesorado de Enseñanzas Técnicas el que en mayor grado lo concibe como un trabajo que integra la aplicación de diversas metodologías y herramientas presentadas en las materias, a la vez que asume como muy relevante su contribución en la preparación para el ejercico profesional y la transferencia de conocimiento. Por su parte, son también los/as 
docentes de las titulaciones de Enseñanzas Técnicas los que tienen una mayor experiencia en la tutorización de trabajos en colaboración con empresas u otras instituciones.

Esta realidad diferencial puede venir determinada, en gran parte, por la experiencia previa con que contaba el profesorado de las titulaciones de ingeniería en relación a los trabajos fin de carrera. En efecto, la implementación del TFG ha dado lugar a dos realidades muy diferentes: por un lado, la de aquellas titulaciones que han visto en esta materia una continuidad con los proyectos fin de carrera y, por otro, la de las que han debido acoger y adaptar el TFG a la estructura de sus planes de estudios.

Pese a estas diferencias, el conjunto del profesorado valora positivamente la elaboración del TFG para la formación del alumnado, al igual que se recoge en otros estudios (Feather, Anchor y Cowton, 2014). La percepción sobre esta contribución varía en función de la modalidad de trabajo fin de grado y el área de conocimiento, reflejo nuevamente de las particulares características de las diferentes áreas y titulaciones.

La mayoría de profesorado encuestado considera que el alumnado no está preparado para enfrentar con autonomía el desarrollo del TFG, lo que contrasta con la valoración dada por el alumnado en otros estudios en los que se mostraban mayoritariamente preparados para su realización (Vera y Briones, 2016). Esta realidad pone el foco de atención en la necesidad de formular acciones específicas de formación que doten al alumnado de estrategias esenciales para el desarrollo, con el mayor grado de autonomía posible, de un trabajo de estas características. Así se está ya implementando en algunos centros de la USC, en línea con lo realizado en otras universidades (Rekalde, 2011).

Esta formación específica puede ser un elemento clave en la disminución de un posible desajuste entre los contenidos trabajados a lo largo de los estudios de grado y las competencias demandadas por la materia de TFG, a la vez que permitiría aligerar la carga de trabajo que asume habitualmente el tutor/a en el asesoramiento sobre cuestiones básicas referentes a la búsqueda de información, citación de fuentes, etc. En este contexto, el tutor está en disposición de dedicar más tiempo a cuestiones esenciales en el proceso de dirección de un trabajo de estas caracterísiticas tales como contribuir a delimitar la pregunta de investigación, que ha sido identificada en otros estudios (Tood, Bannister y Clegg, 2004) como una de las dificultades con que se encuentra el alumnado.

\section{Referencias}

Ávila, M. y Luján, A. L. (2011). Diseño de un sistema de coordinación para el desarrollo, integración y evaluación de competencias genéricas en los títulos de Grado de la Facultad de Educación de Cuenca. En M. Marín, A. B. Morales y D. Delgado (Eds.), Trabajos fin de Grado y Máster: La evaluación global (pp. 183-190). Toledo: Universidad de Castilla La Mancha.

Castro, L., Prats, M. y Arànega, S. (2013). Percepciones de alumnos/as y tutores/as sobre el proceso de acción tutorial en el Trabajo de Final de Máster. Un estudio de caso en la Universidad de Barcelona. Revista Iberoamericana para la Investigación y el Desarrollo Educativo, $10 . \quad$ Recuperado de: http://ride.org.mx/111/index.php/RIDESECUNDARIO/article/view/171/166

Civila, A. (2014). Gestión de unos 800 trabajos fin de grado en una Facultad de Ciencias de la Educación. En K. Pérez, B. Bilbao, E. Fernández de Larrea, B. Otero y P. Ruiz de Gauna (Coord.), Retos y oportunidades del TFG para la sociedad del conocimiento 
(Libro de Actas del I Congreso Interuniversitario del Trabajo Fin de Grado) (pp. 356365). Bilbao: Universidad del País Vasco.

Cohen, L. y Manion, L. (2002). Métodos de Investigación educativa ( $2^{a}$ ed.). Madrid: La Muralla.

Elías, A. (2012). Algunas reflexiones en clave de gestión tras las primeras defensas de los Trabajos Fin de Grado, Revista andaluza de relaciones laborales, 25-26, 113-134. Recuperado http://www.uhu.es/publicaciones/ojs/index.php/trabajo/article/view/2112

Feather, D., Anchor, J.R. y Cowton, C.J. (2014). Supervisors' perceptions of the value of the undergraduate dissertation. The International Journal of Management Education, 12, 14-21.

Fuertes, M.T. y Balaguer, M.C. (2012). El TFG como elemento de mejora de la calidad en la evaluación del módulo practicum. Propuesta de la Universitat Internacional de Catalunya. REDU: Revista de Docencia Universitaria, 10 (2), 329-343. DOI: http://dx.doi.org/10.4995/redu.2012.6111

González, G., Agulló, V. y González, A. (2013). Tutorial multimedia para guiar la preparación y defensa del Trabajo Fin de Grado. @tic, Revista d'innovació educativa, 11, 8-17. DOI: 10.7203/attic.11.3059

Greenbank, P. y Penketh, C. (2009). Student autonomy and reflections on researching and writing the undergraduate dissertation. Journal of Further and Higher Education, 33 (4), 463-472.

Hernández, D., Moreno, V., Camps, I.P., Clarisó, R. y Martínez, A. (2013). Implementación de buenas prácticas en los Trabajos de Fin de Grado. REDU: Revista de Docencia Universitaria, $11 \quad$ ( ${ }^{\circ}$ especial), 269-278. Recuperado de: http://polipapers.upv.es/index.php/REDU/article/view/5556/5547

Jamieson, S., y Gray, C. (2006). The supervision of undergraduate research students: Expectations of student and supervisor. Practice and Evidence of Scholarship of Teaching and Learning in Higher Education, 1(1), 37-59.

Maiztegui, C., Martínez, I., Santibáñez, R. y Martínez, S. (2012). Diseño e implantación del Trabajo Fin de Grado en Educación Social. Análisis de un proceso. En S. Morales, J. Lirio y R. Marí, La Pedagogía Social en la Universidad: Investigación, Formación y Compromiso Social (Seminario Interuniversitario de Pedagogía Social) (pp. 403-416). Valencia: Nau Llibres.

Malcolm, M. (2012). Examining the implications of learner and supervisor perceptions of undergraduate dissertation research in Business and Manegement. Teaching in Higher Education, 11 (5), 565-576.

Martínez, M. T. y Pastor, E. (2014). El Trabajo Fin de Grado en la Facultad de Trabajo Social de la Universidad de Murcia. Azarbe: Revista Internacional de Trabajo Social y Bienestar, 3, 83-91. Recuperado de: http://revistas.um.es/azarbe/article/view/198401

Mateo, J., Escofet, A., Martínez, F., Ventura, J. y Vlachopoulos, D. (2012). The Final year Project (FYP) in social sciencies: establishment of its associated competences and evaluation standards. Studies in Educational Evaluation, 38, 28-34. DOI: https://doi.org/10.1016/j.stueduc.2011.12.002 
Onieva, J.L. (2016). Estrategias didácticas y recomendaciones para la defensa oral de los Trabajos Fin de Grado y Fin de Máster. Revista Electrónica Interuniversitaria de Formación del Profesorado, 19(3), 185-198.

Pascual, M. y Pascual, M. M. (2012). Propuesta de evaluación del Trabajo Fin de Grado en Derecho. Aula Abierta, 40 (1), 85-102. Recuperado de: http://digibuo.uniovi.es/dspace/bitstream/10651/21155/1/AulaAbierta.2012.40.1.85102.pdf

Puigcerver, M.C., Martín, P.J. y Renart, M. (2013). Los beneficios de la coordinación horizontal en la realización de los TFG para los alumnos de Grado en Administración y Dirección de Empresas. Revista de Investigación en Educación, 11 (1), 97-117. Recuperado de: http://reined.webs.uvigo.es/ojs/index.php/reined/article/view/603

Real Decreto 1393/2007, que establece la ordenación de las enseñanzas oficiales de Grado y Master en España. Boletín Oficial del Estado, núm. 260 de 30 de octubre.

Real Decreto 861/2010, por el que se modifica el Real Decreto 1393/2007, de 29 de octubre, por el que se establece la ordentación de las enseñanzas universitarias oficiales. Boletín Oficial del Estado, núm. 161 de 3 de julio.

Rekalde, I. (2011). ¿Cómo afrontar el Trabajo Fin de Grado? Un problema o una oportunidad para culminar con el desarrollo de las competencias. Revista Complutense de Educación, $22 \quad$ (2), 179-193. DOI: http://dx.doi.org/10.5209/rev_RCED.2011.v22.n2.38488

Reyes-García, C.I. y Díaz-Megolla, A. (2017). ¿Se adecúa la normativa del Trabajo Fin de Grado al enfoque de evaluación del Espacio Europeo de Educación Superior? Revista Complutense de Educación, 28(4), 1285-1302.

Rullán, M., Fernández, M., Estapè, G. y Márquez, M. D. (2010). La evaluación de competencias transversales en la materia trabajos fin de grado. Un estudio preliminar sobre la necesidad y oportunidad de establecer medios e instrumentos por ramas de conocimiento. REDU. Revista de Docencia Universitaria, 8(1), 74. Recuperado de: http://red-u.net/redu/files/journals/1/articles/146/public/146-132-2PB.pdf

Rullán, M. y Estapé, G. (2011). Implementación de los Trabajos Fin de Grado. En M. Marín, A.B. Morales y D. Delgado (Eds.), Trabajos Fin de Grado y Máster: La evaluación global (Libro de Actas VII Intercampus) (pp. 17-26). Toledo: Universidad de Castilla La Mancha.

Sánchez, P. (2013). Trabajo Fin de Grado en Administración y Dirección de Empresas (ADE). De la teoría a la experiencia de la Facultad de CC. Empresariales y Turismo del Campus de Ourense (Universidad de Vigo). REDU: Revista de Docencia Universitaria, 11 (3), 461-481. DOI: http://dx.doi.org/10.4995/redu.2013.5539

Tood, M., Bannister, P. y Clegg, S. (2004). Independent inquiry and the undergraduate dissertation: Perceptions and experiences of final-year social science students. Assessment \& Evaluation in Higher Education, 29 (3), 335-355.

Tood, M., Smith, K. y Bannister, P. (2006). Supervising a social science undergraduate dissertation: staff experiences and perceptions. Teaching in Higher Education, 11 (2), 161-173.

Torrado, M. (2004). Estudios tipo encuesta. En R. Bisquerra (Coord.), Metodología de la investigación educativa (pp. 130-147). Madrid: La Muralla. 
USC (2016). Regulamento de matrícula, elaboración e defensa dos traballos fin de grao e fin de máster na Universidade de Santiago de Compostela. Recuperado de: http://www.usc.es/gl/normativa/xestionacademica/index.html

Valderrama, E., Rullán, M., Sánchez, F., Pons, J., Cores, M.F. y Bisbal, J. (2009). La evaluación de competencias en los Trabajos Fin de Estudios. En I. Jacob y D. López (Eds.), Actas de las XV Jornadas de Enseñanza Universitaria en Informática (pp. 405-412). Barcelona.

Vera, J. y Briones, E. (2015). Students' perspectives on the processes of supervision and assessment of undergraduate dissertations. Cultura y Educación, 27(4), 726-765. doi:10.1080/11356405.20 15.1089391

Vera, J. y Briones, E. (2016). Un nuevo reto para las universidades: la evaluación de la calidad de la gestión de los Trabajos de Fin de Grado. Foro de Educación, 14(21), 281-310. doi: http://dx.doi.org/10.14516/fde.2016.014.021.014

Vilardell, I. (2010). Experiencia sobre el trabajo de fin de grado en Administración y Dirección de Empresas. Educade, Revista de Educación en Contabilidad, Finanzas y Administración de Empresas, 1(1), 101-122. Recuperado de: http://educade.es/docs/01/07-vilardell.pdf

Zamora, F. y Sánchez, J. (2014). Los Trabajos Fin de Grado: una herramienta de desarrollo de competencias transversales en la Educación Superior. REDU - Revista de Docencia Universitaria, 13(3), 197-211. DOI: http://dx.doi.org/10.4995/redu.2015.5426 